\title{
Results of expectant management in singleton and twin pregnancies complicated by preterm premature rupture of membranes
}

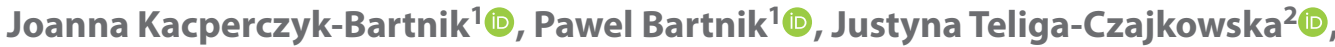 \\ Aneta Malinowska-Polubiec ${ }^{1}{ }^{(1)}$, Agnieszka Dobrowolska-Redo' ${ }^{1}$, \\ Ewa Romejko-Wolniewicz ${ }^{1}$, Patryk Rudzinski $^{3}$, Krzysztof Czajkowski ${ }^{1}$ (D) \\ ${ }^{1} 2^{\text {nd }}$ Department of Obstetrics and Gynecology, Medical University of Warsaw, Poland \\ ${ }^{2}$ Department of Obstetrics and Gynecology Didactics, Medical University of Warsaw, Poland \\ ${ }^{3}$ Students' Scientific Group affiliated to $2^{\text {nd }}$ Department of Obstetrics and Gynecology, Medical University of Warsaw, Poland
}

\begin{abstract}
Objectives: This study aimed to examine whether expectant management in twin pregnancies with preterm premature rupture of membranes ( $P$ PROM) is as safe as in singleton pregnancies.

Material and methods: It was a retrospective cohort study comparing pregnancy course and outcome in singleton $(n=299)$ and twin pregnancies $(n=49)$ complicated by preterm premature rupture of membranes. Analysed factors included maternal diseases, gestational age at premature rupture of membranes (PROM), management during hospitalization, latency periods between PROM and delivery, gestational age at delivery, neonatal management and outcome.

Results: The difference in the proportion of patients with latency up to 72 hours, latency between 72 hours and seven days, and latency exceeding seven days were insignificant. The percentage of patients who received intravenous tocolysis and antenatal corticosteroids were similar; however, patients in twin pregnancies more often received incomplete steroids dose $(p=0.01)$. The occurrence of the positive non-stress test result and signs of intrauterine infection were similar between the groups. No statistically significant differences in the prevalence of neonatal complications except transient tachypnoea of the newborn were identified ( $24 \%$ in the singleton vs $13 \%$ in the twin group, $p=0.03$ ).

Conclusions: Expectant management of pPROM in singleton and twin pregnancies results in similar perinatal and neonatal outcome. Consequently, in case of no evident contraindications, expectant management of twin pregnancies seems to be equally as safe as in singleton pregnancies. Patients in twin pregnancies may be at higher risk of delivery before administration of full antenatal corticosteroids dose, therefore require immediate management initiation and transfer to a tertiary referral centre.
\end{abstract}

Key words: pregnancy outcome; pregnancy; twin; premature birth; preterm premature rupture of the membranes

Ginekologia Polska 2022; 93, 12: 999-1005

\section{INTRODUCTION}

Twin birth rate has been gradually increasing during the recent decades, partly due to pregnancy postponing until more advanced maternal age [1-3]. Availability of infertility treatment methods with ovulation stimulation and assisted reproductive technologies are also associated with more frequent multiple pregnancy occurrences $[4,5]$. The incidence of preterm premature rupture of membranes (pPROM) in twin pregnancies is higher than in singleton pregnancies [6]. Intrauterine treatment of previously incurable condi- tions, including twin-to-twin transfusion syndrome leads to iatrogenic PPROM, which affects up to $50 \%$ of treated cases [7-11]. PPROM management strategies in singleton pregnancies include planned early birth and expectant monitoring [12].

\section{Objectives}

This study aimed to examine whether expectant management in twin pregnancies with PPROM is as safe as in singleton pregnancies. Neonatal complications incidence

\footnotetext{
Corresponding author:

Joanna Kacperczyk-Bartnik

$2^{\text {nd }}$ Department of Obstetrics and Gynecology, Medical University of Warsaw, Poland

e-mail: asiakacperczyk@gmail.com
} 
was compared between twin and singleton PPROM groups managed expectantly.

\section{MATERIAL AND METHODS Patients}

It was a retrospective cohort study comparing pregnancy course and outcome in singleton and twin pregnancies complicated by preterm premature rupture of membranes. We included in our research consecutive patients with rupture of membranes before completed $37^{\text {th }}$ gestational week managed in a tertiary referral centre between October 2016 and December 2018. Exclusion criteria were as follows: patients in multiple pregnancies with more than two foetuses and with congenital foetal anomalies. There were altogether 7198 births during the observation period. Out of them, 299 patients in singleton and 49 in twin pregnancies met the criteria of the study $-4.1 \%$ and $0.7 \%$ of all births, accordingly. In the twin pregnancy group, there were 40 patients in dichorionic diamniotic pregnancies and nine patients in monochorionic diamniotic pregnancies. Analysed factors included maternal diseases, gestational age at PROM, management during hospitalization, latency periods between PROM and delivery, gestational age at delivery, neonatal management and outcome.

\section{Management}

PPROM diagnosis was based on speculum examination, rapid test detecting insulin-like growth factor binding protein (IGFBP-1) and ultrasound scan. Expectant management included empirical antibiotic prophylaxis with intravenous cefuroxime for ten days, non-stress tests, ultrasound examinations, and monitoring of serum inflammatory markers levels. The antibiotic therapy was modified if needed according to the results of each patient's individual cervical culture result and antibiogram collected on admission as soon as it was available. Every two weeks, we repeated the cervical swab and continued personalized antibiotic treatment, if the result was positive. Latency period was identified as the time between PPROM and birth.

\section{Neonatal outcome}

Low birth weight was defined as birth weight below $2500 \mathrm{~g}$, very low birth weight — as birth weight under $1500 \mathrm{~g}$, and extremely low birth weight — as birth weight less than $1000 \mathrm{~g}$. We considered the birth weight below $10^{\text {th }}$ percentile as hypotrophy. Early-onset sepsis (EOS) occurred within 72 hours after birth, whereas late-onset sepsis (LOS) developed after 72 hours after delivery. We diagnosed transient tachypnoea of the newborn and respiratory distress syndrome based on clinical presentation and chest X-ray. Respiratory failure was defined as persistent hypoxemia or hypercapnia despite surfactant therapy and 'maximal' conventional ventilation.

\section{Statistical analysis}

Statistical analysis was performed using Statistica 13 (StatSoft. Inc.): the T-Student test - for quantitative data comparison between two groups and two-sided Fisher's exact test - for discrete variables.

\section{RESULTS \\ Group characteristics}

Mean maternal age was similar between the groups (Tab. 1). There were no differences regarding primiparity. The history of the previous caesarean section more often occurred in singleton pregnancies $(p=0.04)$.

Differences in the incidence of the following features were insignificant: gestational diabetes, pregestational diabetes, hypertension, hypothyroidism, and intrahepatic cholestasis of pregnancy (Tab. 1). There was no difference in the incidence of cervical insufficiency, the need for cervical cerclage or gestational age at PROM (32.8 vs 32.7 weeks) and delivery (33.5 vs 33.2 weeks). The difference in the proportion of patients with latency up to 72 hours, latency between 72 hours and seven days, and latency exceeding seven days were insignificant (Tab. 2).

Differences in the occurrence of the pathogenic culture of cervical specimen collected in all patients and amniotic fluid obtained during caesarean sections were also non-significant (Tab. 1). The percentage of patients who received intravenous tocolysis and antenatal corticosteroids were similar; however, patients in twin pregnancies more often received incomplete steroids dose $(p=0.01)$ (Tab. 3). The occurrence of the positive non-stress test result and signs of intrauterine infection were similar between the groups (Tab. 3). More patients in the twin group delivered by C-section $(p<0.001)$ but the emergency caesarean section rate was comparable (Tab. 3).

\section{Neonatal outcome}

Children born from twin pregnancies had lower mean birth weight $(p<0.001)$ and length $(p=0.049)$. There was no difference regarding the ponderal index between compared groups. No statistically significant differences in the prevalence of neonatal complications except transient tachypnoea of the newborn were identified ( $24 \%$ in the singleton vs $13 \%$ in the twin group, $p=0.03$ ) (Tab. 4). In the singleton group, there was an association between positive maternal cervical culture and more frequent development of early-onset sepsis in newborns ( $p=0.015)$. That was not valid for the twin group. Distribution of early-onset sepsis in children from singleton and twin pregnancies depending on the result of maternal cervical culture is presented in Figures 1 and 2 . 
Table 1. Group characteristics and pregnancy course

\begin{tabular}{|l|c|c|c|}
\hline Feature & Singleton pregnancies $(\mathbf{n}=\mathbf{2 9 9})$ & Twin pregnancies $(\mathbf{n}=\mathbf{4 9})$ & $\mathbf{p}$ \\
\hline Mean maternal age [years] & 32.6 & 31.9 & 0.43 \\
\hline Primiparity (\%) & $132(44)$ & $26(53)$ & 0.28 \\
\hline History of caesarean section (\%) & $69(23)$ & $5(10)$ & 0.04 \\
\hline Cervical insufficiency (\%) & $47(16)$ & $12(24)$ & 0.15 \\
\hline Cervical cerclage (\%) & $10(3)$ & 0 & 0.36 \\
\hline Gestational diabetes mellitus (\%) & $71(24)$ & $8(16)$ & 0.27 \\
\hline Pregestational diabetes mellitus (\%) & $20(7)$ & $2(4)$ & 0.75 \\
\hline Hypertension (\%) & $24(8)$ & $3(6)$ & 1 \\
\hline Intrahepatic cholestasis of pregnancy (\%) & $9(3)$ & $4(8)$ & 0.09 \\
\hline Hypothyroidism (\%) & $58(19)$ & $14(29)$ & 0.18 \\
\hline Pathogenic cervical culture (\%) & $130(43)$ & $21(43)$ & 1 \\
\hline Pathogenic amniotic fluid culture* (\%) & $19(14)$ & $3(8)$ & 0 \\
\hline Intrauterine foetal demise (\%) & $2(0.7)$ & 0.42 \\
\hline
\end{tabular}

*Amniotic fluid culture was performed only in patients undergoing caesarean section: $n=135$ in singleton pregnancies and $n=40$ in twin pregnancies

Table 2. Gestational age and latency period

\begin{tabular}{|l|c|c|c|}
\hline Feature & Singleton pregnancies, $\mathbf{n = 2 9 9}$ & Twin pregnancies, $\mathbf{n = 4 9}$ & $\mathbf{p}$ \\
\hline Gestational age at PROM [weeks] & 32.8 & 32.7 & 0.84 \\
\hline Gestational age at delivery [weeks] & 33.5 & 33.2 & 0.49 \\
\hline Latency period up to $72 \mathrm{~h}(\%)^{*}$ & $202(68 \%)$ & $39(80 \%)$ & 0.10 \\
\hline Latency period between $72 \mathrm{~h}$ and 7 days (\%)* & $45(15 \%)$ & $6(12 \%)$ & 1 \\
\hline Latency period over 7 days (\%)* & $52(17 \%)$ & $4(8 \%)$ & 0.14 \\
\hline
\end{tabular}

*Latency period between PROM and delivery; PROM — premature rupture of membranes

Table 3. Preterm premature rupture of membranes management

\begin{tabular}{|l|c|c|c|}
\hline Feature & Singleton pregnancies, $\mathbf{n = 2 9 9}$ & Twin pregnancies, $\mathbf{n}=\mathbf{4 9}$ & $\mathbf{p}$ \\
\hline Intravenous tocolysis (\%) & $105(35)$ & $22(45)$ & 0.2 \\
\hline Antenatal corticosteroids, any dose (\%) & $184(62)$ & $36(73)$ & 0.11 \\
\hline Antenatal corticosteroids, incomplete dose (\%) & $35(12)$ & $13(27)$ & 0.01 \\
\hline Antenatal corticosteroids, full dose (\%) & $149(50)$ & $23(47)$ & 0.76 \\
\hline $\begin{array}{l}\text { Birth within } 48 \mathrm{~h}-14 \text { days after antenatal } \\
\text { corticosteroids full dose administration (\%) }\end{array}$ & $61(20)$ & $5(10)$ & 0.12 \\
\hline $\begin{array}{l}\text { Birth after 14 days since antenatal corticosteroids full } \\
\text { dose administration (\%) }\end{array}$ & $27(9 \%)$ & $3(6 \%)$ & 0.59 \\
\hline Positive non-stress test (\%) & $48(16)$ & $3(6)$ & 0.08 \\
\hline Signs of intrauterine infection (\%) & $24(8)$ & $2(4)$ & 0.56 \\
\hline Caesarean section (\%) & $135(45)$ & $40(82)$ & $<0.001$ \\
\hline Non-cephalic presentation, active phase of labor (\%) & $47(16)$ & $35(71)$ & $<0.001$ \\
\hline Emergency caesarean section (\%) & $41(14)$ & $5(10)$ & 0.25 \\
\hline
\end{tabular}

The subgroup analysis concerning neonatal complications rate and duration of the latency period, shown that newborns born from singleton pregnancies within 72 hours after pPROM more frequently developed transient tachypnoea than newborns born from twin pregnancies ( $23.5 \%$ vs $11.5 \%, p=0.02$ ) (Fig. 3 and 4 ). 
Table 4. Comparison of neonatal outcome

\begin{tabular}{|c|c|c|c|}
\hline Feature & $\begin{array}{l}\text { Children born from singleton } \\
\text { pregnancies, } \mathrm{n}=297\end{array}$ & $\begin{array}{l}\text { Children born from twin } \\
\text { pregnancies, } n=98\end{array}$ & p \\
\hline \multicolumn{4}{|l|}{ Birth weight } \\
\hline Mean birth weight [g] & 2416 & 2027 & $<0.001$ \\
\hline Mean length $[\mathrm{cm}]$ & 49.4 & 46.3 & 0.049 \\
\hline Median ponderal index $\left[\mathrm{g} / \mathrm{cm}^{3}\right]$ & 19.5 & 19.7 & 0.88 \\
\hline Low birth weight (\%) & $106(36)$ & $69(70)$ & $<0.001$ \\
\hline Very low birth weight (\%) & $19(6)$ & $13(13)$ & 0.51 \\
\hline Extremely low birth weight (\%) & $13(4)$ & $3(3)$ & 0.77 \\
\hline Birth weight below $10^{\text {th }}$ percentile (\%) & $8(3)$ & $6(6)$ & 0.12 \\
\hline \multicolumn{4}{|l|}{ Respiratory complications } \\
\hline Pulmonary hypoplasia (\%) & $2(0.7)$ & $2(2)$ & 0.29 \\
\hline Respiratory distress syndrome (\%) & $38(13)$ & $12(12)$ & 1 \\
\hline Pneumothorax (\%) & $6(2)$ & 0 & 0.34 \\
\hline Transient tachypnoea of the newborn (\%) & $72(24)$ & $13(13)$ & 0.03 \\
\hline Broncho-pulmonary dysplasia (\%) & $20(7)$ & $7(7)$ & 0.81 \\
\hline Pulmonary hypertension (\%) & $12(4)$ & $1(1)$ & 0.2 \\
\hline Respiratory failure (\%) & $77(26)$ & $25(25)$ & 1 \\
\hline \multicolumn{4}{|l|}{ Infectious complications } \\
\hline Early-onset sepsis (\%) & $23(8)$ & $3(3)$ & 0.15 \\
\hline Late-onset sepsis (\%) & $14(5)$ & $6(6)$ & 0.6 \\
\hline Pneumonia (\%) & $13(4)$ & $6(6)$ & 0.42 \\
\hline \multicolumn{4}{|l|}{ Other complications } \\
\hline Hyperbilirubinemia (\%) & $182(61)$ & $68(68)$ & 0.08 \\
\hline Anaemia (\%) & $49(16)$ & $24(24)$ & 0.07 \\
\hline Hypoglycaemia (\%) & $51(17)$ & $13(13)$ & 0.43 \\
\hline Intraventricular haemorrhage (\%) & $20(7)$ & $3(3)$ & 0.22 \\
\hline Necrotizing enterocolitis (\%) & $5(2)$ & 0 & 0.34 \\
\hline Retinopathy (\%) & $7(2)$ & $3(3)$ & 0.71 \\
\hline Circulatory failure (\%) & $20(7)$ & $2(2)$ & 0.12 \\
\hline Death (\%) & $6(2)$ & $3(3)$ & 0.7 \\
\hline \multicolumn{4}{|l|}{ Management } \\
\hline NICU admission (\%) & $76(26)$ & $21(21)$ & 0.5 \\
\hline Mechanical ventilation (\%) & $46(15)$ & $8(8)$ & 0.09 \\
\hline nCPAP* $(\%)$ & $100(34)$ & $33(33)$ & 1 \\
\hline Mean hospitalization duration [days] & 22.34 & 20.88 & 0.55 \\
\hline
\end{tabular}

*Neonatal Continuous Positive Airway Pressure

\section{DISCUSSION}

Overall, we identified more similarities than differences between analysed groups. One of the differences regarding the neonatal outcome in singleton and twin pregnancies complicated by pPROM was mean birth weight, mean length and the occurrence of low birth weight. These findings, however, are not directly associated with the course of PPROM.
It is known that neonates born from multiple gestations have constitutionally lower birth weight than children from singleton pregnancies [13-15]. The incidence of neonatal birth weight below $10^{\text {th }}$ percentile and difference in the median ponderal index were insignificant. The pregnancy course in singleton and twin pregnancies in this study was characterized by similar mean gestational age at PROM 
and delivery. Patients in the twin group more often received incomplete antenatal corticosteroids dose. However, the percentage of patients who delivered between 48 hours and 14 days since the completion of antenatal corticosteroids dose was similar in both groups. We did not observe

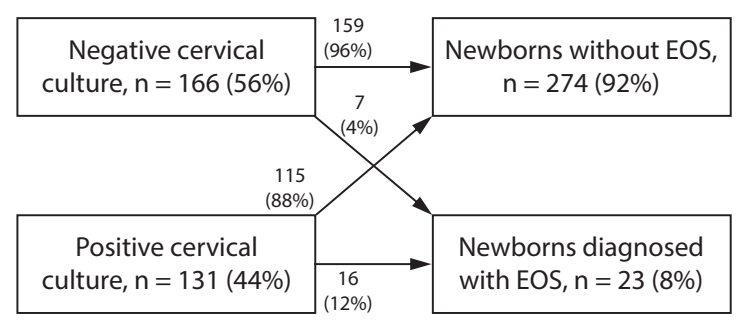

Figure 1. Association between maternal cervical culture results and the occurrence of early-onset sepsis in newborns from singleton pregnancies; $p=0.015$; EOS - early-onset sepsis

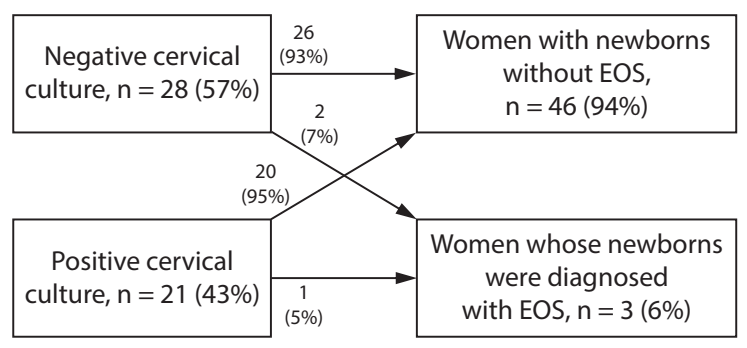

Figure 2. Association between maternal cervical culture results and the occurrence of early-onset sepsis in newborns from twin pregnancies; $p=1$; EOS - early-onset sepsis any differences in the occurrence of the following neonatal complications: pulmonary hypoplasia, respiratory distress syndrome, pneumothorax, broncho-pulmonary dysplasia, pulmonary hypertension, respiratory failure, use of mechanical ventilation, nor use of neonatal continuous positive airway pressure (nCPAP). There was a higher prevalence of transient tachypnoea of the newborn in the singleton group. The reason for it could be a difference in indications for the caesarean section between the groups. Because of more frequent non-cephalic presentation in the twin group, higher percentage of patients underwent caesarean section during the early active phase of labour, which decreases the risk of transient tachypnoea of the newborn [16].

In a retrospective cohort study by Bianco et al. comparing the outcome of 116 singletons and 116 twin pregnancies complicated by PPROM and managed expectantly, a decreased median latency period in the second group was observed ( 19.5 hours vs 11.5 hours) [17]. Authors reported no other differences in perinatal nor neonatal outcomes. Twin gestation was also one of the factors influencing latency period in cases of PPROM analysed in a retrospective study ( $n=303$ ) by Dagklis et al. [18]. The mean interval between pPROM and delivery was 5.5 and 3.3 days for singleton and twin pregnancies, respectively. In another retrospective case-control study comparing the results of expectant management in PPROM, the median latency period in twin pregnancies was significantly shorter than in singleton gestations, lasting 19 vs 47 hours $(p=0.01)$ [19]. Ekin et al. [20] obtained similar results in a cohort study from Turkey analysing the outcome of 3257 pregnancies complicated

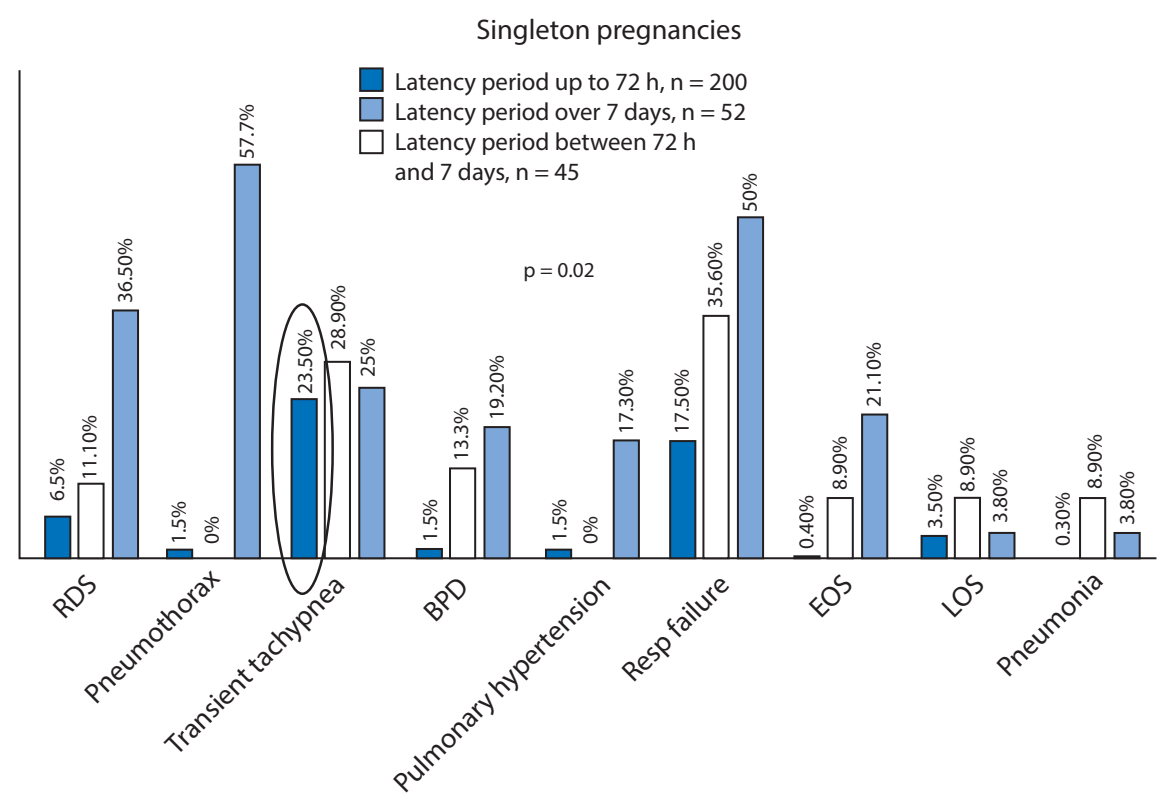

Figure 3. Association between latency periods and the occurrence of neonatal complications in singleton pregnancies; BPD — bronchopulmonary dysplasia; EOS — early-onset sepsis; LOS — late-onset sepsis; RDS — respiratory distress syndrome 


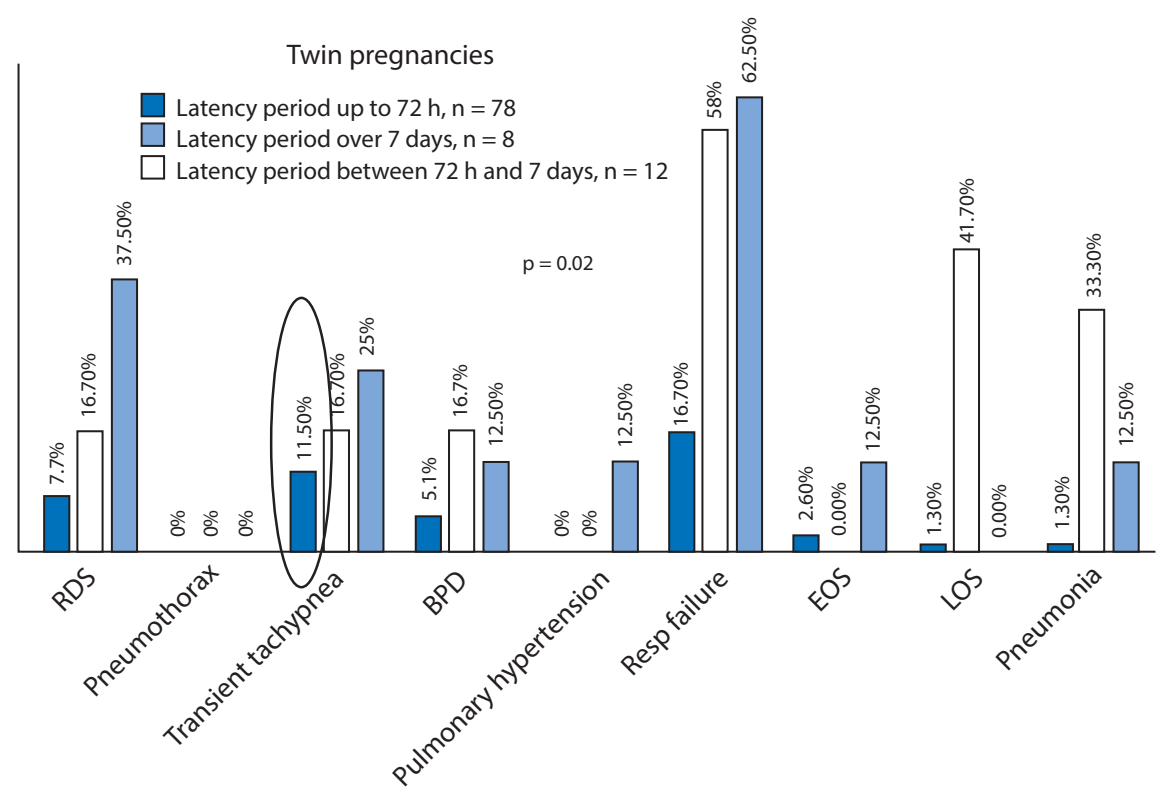

Figure 4. Association between latency periods and the occurrence of neonatal complications in twin pregnancies; BPD —-bronchopulmonary dysplasia; EOS — early-onset sepsis; LOS — late-onset sepsis; RDS — respiratory distress syndrome

by PPROM between 24 and 34 gestational weeks. All mentioned studies focused on the comparison of median latency periods between $\mathrm{PPROM}$ and delivery. In our research, more emphasis is put on the completion of antenatal corticosteroids dosage, rather than on the median latency period alone. We analyzed the percentage of patients who delivered in less than 72 hours following PPROM after receiving incomplete corticosteroids dose and patients who gave birth between 48 hours and 14 days since the completion of antenatal corticosteroids. No difference in the occurrence of delivery within 72 hours from pPROM was identified (Tab. 2). However, a higher proportion of patients who delivered before receiving the complete corticosteroids dose was observed in the twin group (Tab. 3).

In a study by Ehsanipoor et al. [21], the difference in latency period was not statistically significant (median 3.6 days in twin and median 6.2 days in singleton pregnancies, $p=0.86)$. The prevalence of infections among newborns from twin pregnancies analysed in this study was lower than in children from singleton pregnancies (9.8\% vs $23.2 \%$ ). Our observations confirmed the higher prevalence of neonatal early-onset sepsis in association with positive maternal cervical culture within the singleton group (Fig. 1), which was insignificant in the twin group (Fig. 2).

Regarding the neonatal complications, our study did not determine any crucial differences between singleton and twin pregnancies. Other authors reported similar findings $[17,19,22]$. In a study by Kibel et al. [23] comparing singleton and twin pregnancies with PPROM, twin pregnancies were complicated by rupture of membranes at more advanced gestational age ( 29.1 vs 28.5 weeks, $p=0.03)$, had shorter latency period ( 5.0 vs 7.0 days, $p=0.01$ ) and a lower rate of chorioamnionitis. In our study, both the gestational age at PROM and the prevalence of intrauterine infection symptoms, early-onset sepsis, late-onset sepsis, and neonatal pneumonia were similar among singleton and twin pregnancies. According to Trentacoste et al. [24], in the case of twin pregnancies, the infection can be the consequence rather than the cause of pPROM. Antibiotics administration in gestations complicated by PPROM is considered beneficial both as prevention of intrauterine infections and as the reduction in prematurity due to pregnancy prolongation [25-27]. However, data on antibiotics use in twin pregnancies complicated by PPROM is limited. In a randomized multicentre trial ORACLE I, Kenyon et al. [25] did not observe any differences in the latency duration nor occurrence of neonatal positive blood culture between antibiotic and placebo arms in multiple pregnancies subgroup.

\section{CONCLUSIONS}

To conclude, our observations indicate that expectant management of PPROM in singleton and twin pregnancies results in similar perinatal and neonatal outcome. Consequently, in case of no evident contraindications, expectant management of twin pregnancies seems to be equally safe as in singleton pregnancies.

Patients in twin pregnancies may be at higher risk of delivery before administration of full antenatal corticosteroids dose, therefore require immediate management initiation and transfer to a tertiary referral centre. 


\section{Conflict of interest}

All authors declare no conflict of interest.

\section{REFERENCES}

1. Martin JA, Hamilton BE, Osterman MJ.Three decades of twin births in the United States, 1980-2009. 2012. https://stacks.cdc.gov/view/cdc/11675.

2. Blondel B, Kaminski M. Trends in the occurrence, determinants, and consequences of multiple births. Semin Perinatol. 2002; 26(4): 239-249, doi: 10.1053/sper.2002.34775, indexed in Pubmed: 12211614.

3. Imaizumi Y. Trends of twinning rates in ten countries, 1972-1996. Acta Genet Med Gemellol (Roma). 1997; 46(4): 209-218, doi: 10.1017/s0001566000000441, indexed in Pubmed: 9862009.

4. Reynolds MA, Schieve LA, Martin JA, et al. Trends in multiple births conceived using assisted reproductive technology, United States, 1997-2000. Pediatrics. 2003; 111 (5 Pt 2): 1159-1162, indexed in Pubmed: 12728130.

5. Chandra A, Stephen EH. Infertility service use among U.S. women: 1995 and 2002. Fertil Steril. 2010; 93(3): 725-736, doi: 10.1016/j.fertnstert.2008.10.049, indexed in Pubmed: 19100531.

6. Mercer B, Crocker L, Pierce W, et al. Clinical characteristics and outcome of twin gestation complicated by preterm premature rupture of the membranes. Am J Obstet Gynecol. 1993; 168(5): 1467-1473, doi: 10.1016/s0002-9378(11)90783-2.

7. Maggio L, Carr SR, Watson-Smith D, et al. latrogenic Preterm Premature Rupture of Membranes after Fetoscopic Laser Ablative Surgery. Fetal Diagn Ther. 2015; 38(1): 29-34, doi: 10.1159/000369250, indexed in Pubmed: 25427832

8. Beck $V$, Lewi $P$, Gucciardo $L$, et al. Preterm prelabor rupture of membranes and fetal survival after minimally invasive fetal surgery: a systematic review of the literature. Fetal Diagn Ther. 2012; 31(1): 1-9, doi: 10.1159/000331165, indexed in Pubmed: 22104520.

9. Papanna R, Molina S, Moise KY, et al. Chorioamnion plugging and the risk of preterm premature rupture of membranes after laser surgery in twin-twin transfusion syndrome. Ultrasound Obstet Gynecol. 2010; 35(3): 337-343, doi: 10.1002/uog.7476, indexed in Pubmed: 20054828.

10. Papanna R, Block-Abraham D, Mann LK, et al. Risk factors associated with preterm delivery after fetoscopic laser ablation for twin-twin transfusion syndrome. Ultrasound Obstet Gynecol. 2014; 43(1): 48-53, doi: 10.1002/uog.13206, indexed in Pubmed: 24013922.

11. Stirnemann J, Djaafri F, Kim A, et al. Preterm premature rupture of membranes is a collateral effect of improvement in perinatal outcomes following fetoscopic coagulation of chorionic vessels for twin-twin transfusion syndrome: a retrospective observational study of 1092 cases. BJOG. 2018; 125(9): 1154-1162, doi: 10.1111/1471-0528.15147, indexed in Pubmed: 29380497.

12. Buchanan SL, Crowther CA, Levett KM, et al. Planned early birth versus expectant management for women with preterm prelabour rupture of membranes prior to 37 weeks' gestation for improving pregnancy outcome. Cochrane Database Syst Rev. 2010(3): CD004735, doi: 10.1002/14651858.CD004735.pub3, indexed in Pubmed: 20238332.

13. Li Z, Umstad MP, Hilder L, et al. Australian national birthweight percentiles by sex and gestational age for twins, 2001-2010. BMC Pediatr. 2015; 15: 148, doi: 10.1186/s12887-015-0464-y, indexed in Pubmed: 26450410.
14. ANANTH C, VINTZILEOS A, SHEN-SCHWARZ S, et al. Standards of Birth Weight in Twin Gestations Stratified by Placental Chorionicity. Obstetrics \& Gynecology. 1998; 91(6): 917-924, doi: 10.1097/00006250199806000-00008.

15. Glinianaia SV, Skjaerven R, Magnus P. Birthweight percentiles by gestational age in multiple births. A population-based study of Norwegian twins and triplets. Acta Obstet Gynecol Scand. 2000; 79(6): 450-458, indexed in Pubmed: 10857868.

16. Derbent A, Tatli MM, Duran M, et al. Transient tachypnea of the newborn: effects of labor and delivery type in term and preterm pregnancies. Arch Gynecol Obstet. 2011;283(5): 947-951, doi: 10.1007/s00404-010-1473-6, indexed in Pubmed: 20431892.

17. Bianco AT, Stone J, Lapinski $R$, et al. The clinical outcome of preterm premature rupture of membranes in twin versus singleton pregnancies. Am J Perinatol. 1996; 13(3): 135-138, doi: 10.1055/s-2007-994310, indexed in Pubmed: 8688101.

18. Dagklis T, Petousis S, Margioula-Siarkou C, et al. Parameters affecting latency period in PPROM cases: a 10-year experience of a single institution. J Matern Fetal Neonatal Med. 2013; 26(14): 1455-1458, doi: 10.3109/14767058.2013.784257, indexed in Pubmed: 23488655

19. Jacquemyn $Y$, Noelmans $L$, Mahieu $L$, et al. Twin versus singleton pregnancy and preterm prelabour rupture of the membranes. Clin Exp Obstet Gynecol. 2003; 30(2-3): 99-102, indexed in Pubmed: 12854852.

20. Ekin A, Gezer C, Taner CE, et al. Risk factors and perinatal outcomes associated with latency in preterm premature rupture of membranes between 24 and 34 weeks of gestation. Arch Gynecol Obstet. 2014; 290(3): 449-455, doi: 10.1007/s00404-014-3227-3, indexed in Pubmed: 24695905.

21. Ehsanipoor RM, Arora N, Lagrew DC, et al. Twin versus singleton pregnancies complicated by preterm premature rupture of membranes. J Matern Fetal Neonatal Med. 2012; 25(6): 658-661, doi: 10.3109/14767058.2011.584924, indexed in Pubmed: 21736498.

22. Esin S, Gyimadu A, Atak Z, et al. Preterm premature rupture of membranes in singleton vs twin pregnancies: The latency periods and the clinical outcomes revisited. J Obstet Gynaecol. 2014; 34(7): 593-597, doi: 10.3109/01443615.2014.920781, indexed in Pubmed: 24910914.

23. Kibel M, Barrett J, Tward C, et al. The natural history of preterm premature rupture of membranes in twin pregnancies. J Matern Fetal Neonatal Med. 2017; 30(15): 1829-1835, doi: 10.1080/14767058.2016.1228052, indexed in Pubmed: 27550343.

24. Trentacoste SV, Jean-Pierre C, Baergen $R$, et al. Outcomes of preterm premature rupture of membranes in twin pregnancies. J Matern Fetal Neonatal Med. 2008; 21(8):555-557, doi: 10.1080/14767050802131929, indexed in Pubmed: 18609351

25. Kenyon SL, Taylor DJ, Tarnow-Mordi W. Broad-spectrum antibiotics for preterm, prelabour rupture of fetal membranes: the ORACLE I randomised trial. The Lancet. 2001; 357(9261): 979-988, doi: 10.1016/s01406736(00)04233-1.

26. Kenyon S, Pike K, Jones DR, et al. Childhood outcomes after prescription of antibiotics to pregnant women with preterm rupture of the membranes: 7-year follow-up of the ORACLE I trial. The Lancet. 2008; 372(9646): 1310-1318, doi: 10.1016/s0140-6736(08)61202-7.

27. Sela HY, Simpson LL. Preterm premature rupture of membranes complicating twin pregnancy: management considerations. Clin Obstet Gynecol. 2011; 54(2): 321-329, doi: 10.1097/GRF.0b013e318217d60d, indexed in Pubmed: 21508702 